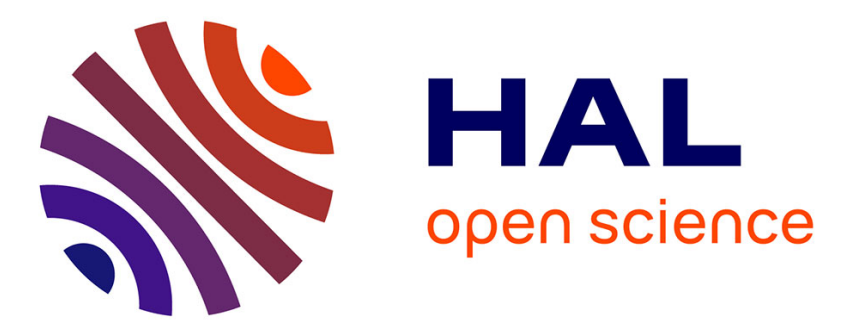

\title{
Matériaux pour l'enregistrement magnétique haute densité
}

S. Sikorav

\section{To cite this version:}

S. Sikorav. Matériaux pour l'enregistrement magnétique haute densité. Revue de Physique Appliquée, 1986, 21 (11), pp.623-633. 10.1051/rphysap:019860021011062300 . jpa-00245482

\section{HAL Id: jpa-00245482 https://hal.science/jpa-00245482}

Submitted on 1 Jan 1986

HAL is a multi-disciplinary open access archive for the deposit and dissemination of scientific research documents, whether they are published or not. The documents may come from teaching and research institutions in France or abroad, or from public or private research centers.
L'archive ouverte pluridisciplinaire HAL, est destinée au dépôt et à la diffusion de documents scientifiques de niveau recherche, publiés ou non, émanant des établissements d'enseignement et de recherche français ou étrangers, des laboratoires publics ou privés. 


\title{
REVUE DE PHYSIQUE APPLIQUÉE
}

Revue Phys. Appl. 21 (1986) 623-633

NOVEMBRE 1986, PAGE 623

Classification

Physics Abstracts

75-70

\section{Matériaux pour l'enregistrement magnétique haute densité}

\author{
S. Sikorav \\ Rhône Poulenc Recherches, 14 rue des Gardinoux, 93300 Aubervilliers, France
}

(Reçu le 20 février 1986, accepté le 26 mai 1986)

\begin{abstract}
Résumé. - Les prochaines générations de media mettront en jeu des densités d'information beaucoup plus importantes que celles actuellement utilisées : accroissement des densités linéaires d'un facteur 3 à 5 , des densités radiales d'un facteur 2 à 3 . Diverses technologies (enregistrement magnétique sur couche particulaire ou sur film mince, enregistrement magnétooptique ou optique réversible) sont potentiellement capables de satisfaire ces exigences, mais c'est l'enregistrement magnétique sur couche particulaire qui paraît le candidat le mieux placé du point de vue pratique, en raison d'une part de l'acquis (moyens de production éprouvés, performances et flexibilité d'emploi des produits) et d'autre part de ses capacités non encore exploitées (passage du mode d'enregistrement longitudinal aux modes vertical et isotrope, évolution des particules, amélioration des procédés de dispersion, enduction et finition). L'objet de cette présentation est de montrer comment les problèmes liés à l'enregistrement haute densité (résolution, rapport signal/bruit ...) gouvernent l'évolution en matière de particules : hausse de la coercivité et de l'aimantation spécifique par unité de volume, tendance à des particules de plus en plus fines (tendance limitée par le phénomène de superparamagnétisme) contrôle de la taille, de la morphologie, de l'homodispersité et de l'état de surface, particules se prêtant particulièrement bien à l'enregistrement isotrope ou vertical (particules isotropes et/ou à anisotropie multiaxiale, plaquettes d'hexaferrite de baryum). L'accent est mis sur la nécessité de prendre en compte les problèmes de stabilité chimique et de sensibilité vis-à-vis des contraintes thermiques et mécaniques, problèmes non encore résolus sur les deux types de particules largement utilisées que sont le fer métal et les oxydes dopés cobalt.
\end{abstract}

\begin{abstract}
The next generations of media will use recording densities much greater than those in use today : linear densities will be multiplied by a factor of 3 to 5 and radial densities by a factor of 2 to 3 . Different technologies (particulate or thin film recording, optics or magnetooptics), are theoretically capable of meeting these requirements, but among them it is the particulate magnetic recording which is the most suitable : first because of its present qualities (tested production methods, performance and flexibility of use), and second, because of its as yet unexplored capabilities (transition from the longitudinal mode to the vertical or isotropic one, evolution of particles, improvement of dispersion, enduction and finishing processes). The purpose of this review is to show how the constraints of high density recording (resolution, signal to noise ratio...) will condition the evolution of particles : higher coercivity and magnetization by volume unit, trend towards smaller particles (limited by the phenomenon of superparamagnetism), better control of the size, morphology, homodispersity and surface aspect, isotropic particles with multiaxial anisotropy, baryum hexaferrites. A special emphasis is laid on the need to take account of the problems of temperature and stress dependence of the cobalt doped iron oxides and of chemical stability of iron particles.
\end{abstract}

\section{Introduction.}

Avec le développement des techniques de la communication et de l'électronique, s'est accrue l'importance des média magnétiques. Les densités d'information contenues dans ces différents media n'ont cessé au cours des dix dernières années d'augmenter et des valeurs de 1 à $2 \times 10^{6}$ bits $/ \mathrm{cm}^{2}$ sont devenues relativement courantes. Les figures 1 et 2 illustrent cette évolution dans les domaines particuliers de la vidéo (analogique) et des disques souples pour informatique (on notera la différence d'échelle qui 


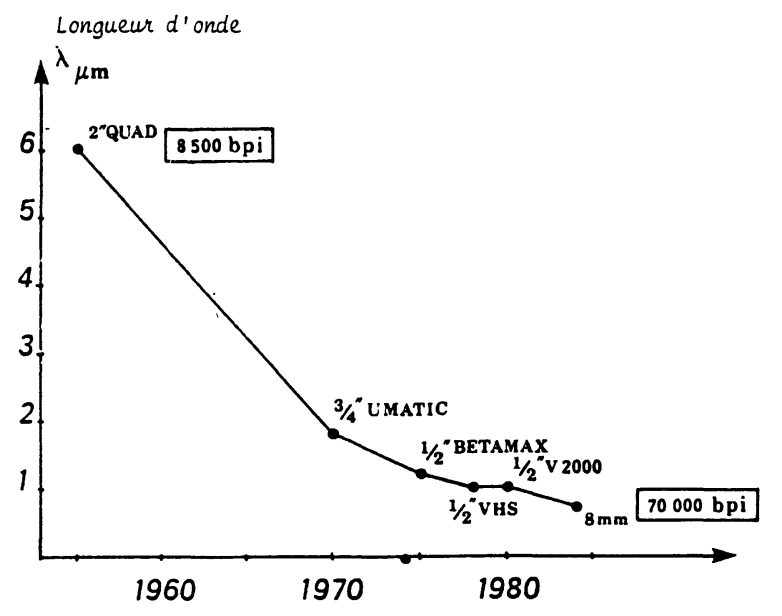

Fig. 1. - L'évolution des densités en vidéo.

[Evolution of densities in video recording.]

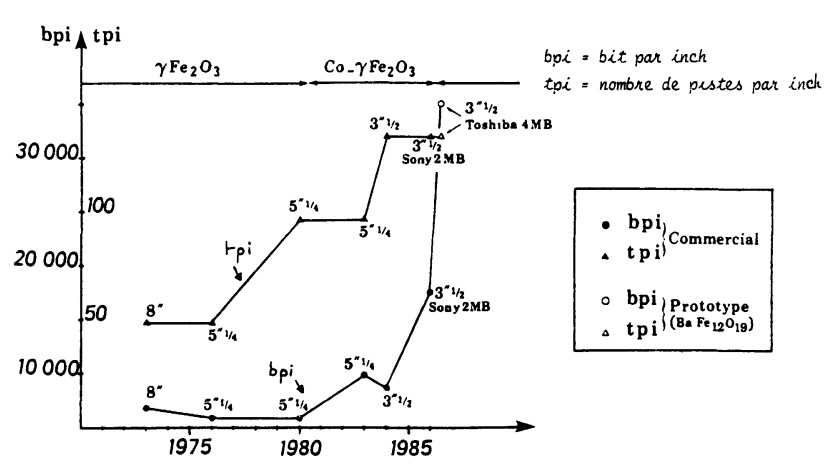

Fig. 2. - L'évolution des densités en floppy disque.

[Evolution of densities in floppy disks.]

existe entre les densités utilisées dans ces deux applications ; des valeurs équivalentes à $70000 \mathrm{bpi}$ ${ }^{1}$ ) étant pratiquement atteintes en vidéo $8 \mathrm{~mm}$, alors que la densité des disques souples 3" $1 / 2600 \mathrm{Oe}$ n'est aujourd'hui que de $8500 \mathrm{bpi}$, décalage dû aux contraintes de « taux d'erreur nul » inhérentes aux applications en informatique).

L'évolution des systèmes, la tendance à la miniaturisation des équipements, la diminution des temps d'accès et l'accroissement des capacités de stockage, le passage progressif du mode analogique au mode digital (possibilité de correction d'erreur et suppression des problèmes de bruit), font que de nouvelles augmentations des densités d'enregistrement sont encore nécessaires.

A l'heure actuelle, l'essentiel des supports d'enregistrement produits met en œuvre l'enregistrement magnétique sur couche particulaire : des particules ferro ou ferrimagnétiques sont dispersées dans une matrice liante, l'enduit formé est déposé par la suite sur un support souple ou rigide, l'axe de facile aimantation des particules, orientées ou non, étant

( $\left.{ }^{1}\right)$ bpi : bit par inch. situé dans le plan de la couche (enregistrement longitudinal, cf. Fig. 3). La figure 4 montre l'aspect en coupes longitudinale et transversale d'une bande pour calculateur (media orienté).

La question qui se pose est de savoir quelles sont les technologies qui pourront d'ici cinq ans permettre les accroissements de densité désirés et quelle est parmi elles, la place de l'enregistrement magnétique particulaire. A priori, trois voies sont ouvertes [1] :
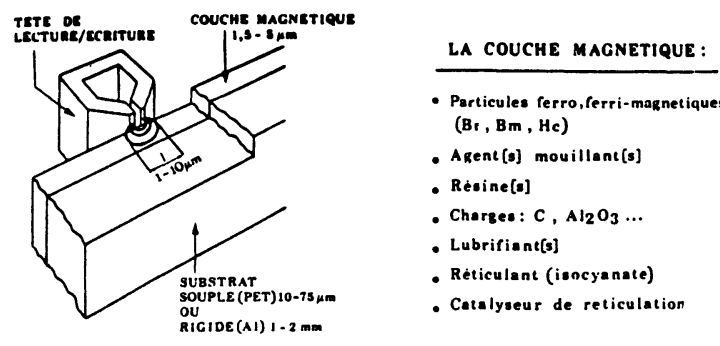

Fig. 3. - L'enregistremen magnétique particulaire.

[The particulate magnetic recording.]

1.1 L'ENREGISTREMENT MAGNETIQUE SUR COUCHE MINCE, longitudinal (ex. couches Co-P de AMPEX) ou perpendiculaire (ex. Co-Cr [2]), très prometteur lors de l'annonce des premiers prototypes voici quelques années, ne connaît pas le développement espéré : les problèmes rencontrés se situent tant au niveau du produit (tenue insuffisante des films vis-à-vis de la corrosion, faible endurance sous tête), que du procédé (prix de revient et investissements trop élevés). En outre, la technique couche mince, bien adaptée à la production de disques, est difficilement envisageable pour la production en masse de bandes.

1.2 L'ENREGISTREMENT MAGNETIQUE SUR COUCHE PARTICULAIRE, déjà le plus employé à l'heure actuelle, bénéficie pour son développement vers les hautes densités des problèmes rencontrés par l'enregistrement magnétique sur film mince. Ses performances peuvent être encore grandement améliorées, la démarche étant, soit :

- de repousser les limites de l'enregistrement longitudinal par l'emploi de particules orientées de très haute coercivité (choix des particules de fer pour la vidéo $8 \mathrm{~mm}$ ),

- soit d'exploiter la composante verticale du champ délivré par la tête d'écriture (composante d'autant plus importante par rapport à la composante longitudinale que la densité est plus élevée) par utilisation de milieux particulaires supportant une large composante d'aimantation perpendiculaire. C'est l'intérêt des particules isotropes à anisotropie multiaxiale $[4,5]$ et des hexaferrites de baryum récemment développées (axe de facile aimantation perpendiculaire au plan des plaquettes) [5].

1.3 LES TECHNIQUES OPTIQUES ET MAGNETOOPTIQUES permettent des densités théoriques encore plus 


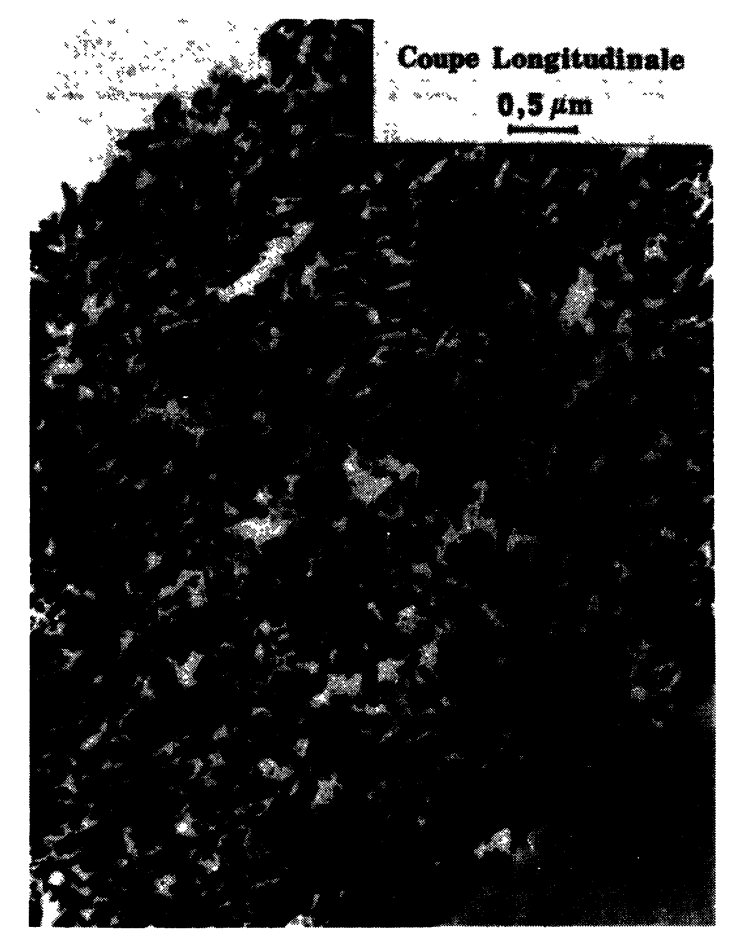

Fig. 4. - M.E.T. en coupe d'une bande calculateur.

[Cross section of a computer tape (T.E.M.).]

élevées que celles évoquées en enregistrement magnétique sur film mince ou sur couche particulaire (les ordres de grandeur avancés sont de $10^{8} \mathrm{bits} / \mathrm{cm}^{2}$ à taux d'erreur nul) et le principe de lecture optique résout les problèmes d'endurance dus au contact tête-couche dans les systèmes magnétiques classiques. Les systèmes optiques ont cependant pour inconvénient d'être dépendants des technologies couches minces (cas des couches optiques Te-Se ou magnétooptiques GdTeTb).

On peut raisonnablement supposer que les techniques de dépôt sous vide sur disque ont plus de chances d'être développées pour les systèmes les plus performants que sont les systèmes magnétooptiques que pour l'enregistrement magnétique sur couche mince. Dans ce scénario, les techniques optiques pourraient commencer à concurrencer sérieusement l'enregistrement magnétique particulaire d'ici une dizaine d'années.

\section{L'enregistrement magnétique sur couche particu-} laire : l'évolution des particules.

L'enregistrement magnétique sur couche particulaire est donc la seule technologie qui puisse, raisonnablement, fournir les produits haute densité des cinq-dix ans à venir : quelle doit donc être conjointement l'évolution des particules magnétiques?

La figure 5 nous montre quelle est la relation entre les performances recherchées sur le produit fini, et les propriétés des particules qui servent à son élaboration.

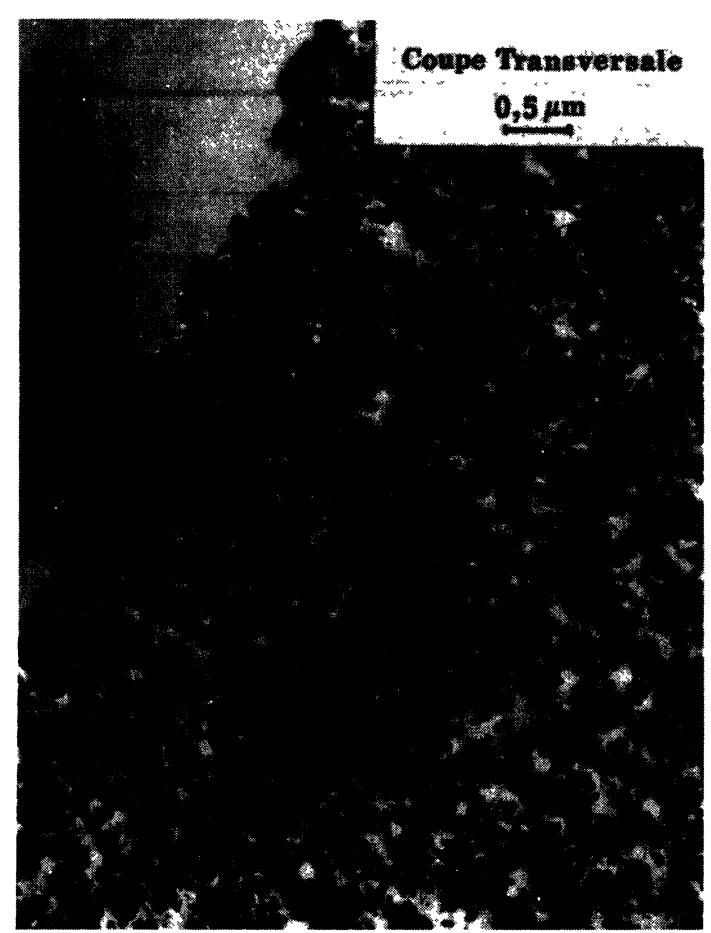

En résumé, on retiendra que les particules de demain seront :

- de plus en plus fines (voisines de $0,1 \mu \mathrm{m}$ ),

- uniformes en taille, morphologie et dopage,

- de forts champ coercitif et aimantation rémanente par unité de volume (ex. particules de fer),

- à anisotropie multiaxiale (ex. dopées cobalt isotropes) ou privilégiant une orientation de l'aimantation perpendiculaire au plan de la couche (hexaferrites de baryum),

- stables chimiquement,

- de caractéristiques magnétiques stables vis-à-vis des contraintes thermiques et mécaniques.

La diminution de taille des particules et donc les performances de l'enregistrement magnétique particulaire, sont limitées par deux phénomènes physiques : le superparamagnétisme i.e. le retournement de l'aimantation sous le seul effet du quantum d'énergie thermique et le « spin canting » i.e. le non alignement des spins situés en surface avec ceux situés dans la masse de la particule. Comme nous le reverrons plus loin, si le phénomène du superparamagnétisme est une contrainte pour tous les types de particules, le spin canting n'a pas lieu pour les particules de fer métal.

Les différentes classes de particules utilisées ou utilisables en enregistrement magnétique sont analysées ci-dessous ; on met en évidence les points à améliorer sur chacune d'elles (variation thermique des propriétés magnétiques des dopés cobalt, passivation du fer ...) et leurs avantages respectifs pour l'enregistrement haute densité. 


\begin{tabular}{|c|c|c|c|c|}
\hline PERFORMANCES MEDIA & $\begin{array}{c}\text { CARACTERISTIQUES PHYSIQUES } \\
\text { MEDIA }\end{array}$ & EKOLUTION DES PARTICULES & $\begin{array}{l}\text { LIMITATIONS } \\
\text { PHYSIQUES } \\
\end{array}$ & $\begin{array}{l}\text { TYPE DE PARTIQULES } \\
\text { FAVORISE }\end{array}$ \\
\hline NIVEAU SORTIE & Br, HC ELEVES & $\sigma_{r}, \rho$, HC ELEVES & $\begin{array}{l}\text { SATURATION TETE } \\
\text { LECTURE }\end{array}$ & FER METAL \\
\hline RAPPORT SIGNAL/BRUIT & $\begin{array}{l}\text { No PARTICULES/UNITE DE } \\
\text { VOLUME ELEVE }\end{array}$ & $\begin{array}{l}\text { PARTICULES FINES } \\
\text { SANS RAMIFICATION } \\
\rightarrow \text { COMPACTAGE }\end{array}$ & $\begin{array}{l}\text { SUPERPARAMAGNETISME } \\
\text { SPIN CANTING }\end{array}$ & FER METAL \\
\hline RESOLUTION & $\begin{array}{l}\text { - HC ELEVE } \\
\text { - EPAISSEUR FAIBLE (emr. long) } \\
\text { - MILIEU A AIMANTATION } \\
\text { ISOTROPE OU PERPENDICU- } \\
\text { LAIRE }\end{array}$ & $\begin{array}{l}\text { HC ELEVE } \\
\text { PARTICULE A ANTSOTROPIE MULTIAXXIALE } \\
\text { OU HEXAFERRITES }\end{array}$ & $\begin{array}{l}\text { SATURATION OES } \\
\text { TETES DE LECTURE } \\
\text { (TETES UNIPOLATRES) }\end{array}$ & $\begin{array}{l}\text { FER METAL } \\
\gamma_{\mathrm{Fe}_{2} \mathrm{O}_{3}-\mathrm{CO}} \\
\text { ISOTROPE } \\
\text { HEXAFERRITES }\end{array}$ \\
\hline $\begin{array}{l}\text { SURECRITURE (DISQUE) } \\
\text { PEAK SHIFT (BANDE) }\end{array}$ & $\begin{array}{l}\text { - SWITCHING FIELD DISTRI- } \\
\text { BUTION } \\
\text { ISFD }=\frac{\Delta H C}{H C} \text { । } \\
\text { - FAIBLE EPAISSEUR }\end{array}$ & $\begin{array}{l}\text { POPULATION HOMODISPERSE EN TAILLE, } \\
\text { FORME ET DOPAGE }\end{array}$ & & \\
\hline $\begin{array}{l}\text { STABILITE CHIMIQUE } \\
\text { STABILITE/TEMPERATURE } \\
\text { STABILITE/CONTRAINTES } \\
\text { MECANIQUES } \\
\text { (perte de niveau = } \\
\text { o (passages) }\end{array}$ & . & $\begin{array}{l}\text { PASSIVATION DU FER METAL } \\
\text { LIMITATION : } \\
\text { - DE LA VARIATION DE L'ANISOTROPIE } \\
\text { AVEC T (hexaferrites, } \gamma \mathrm{Fe}_{2} \mathrm{O}_{3} \text {-CO } \\
\text { isotrope) } \\
\text { - DE LA MAGNETOSTRICTION } \\
\text { (r } \mathrm{Fe}_{2} \mathrm{O}_{3} \text {-Co isotrope) }\end{array}$ & & \\
\hline
\end{tabular}

Fig. 5. - L'évolution technique des particules.

[The technical evolution of particles.]

\section{Les particules magnétiques.}

3.1 L'OXYDE DE FER $\gamma \mathrm{Fe}_{2} \mathrm{O}_{3}$. - Les particules de $\gamma \mathrm{Fe}_{2} \mathrm{O}_{3}$ (maghémite), sont les composés les plus anciennement (années 1930) et les plus fréquemment utilisés en enregistrement magnétique. A l'état " brut ", dans les applications audio ou informatique basse densité, ou comme précurseurs de particules dopées cobalt ou de fer métal, elles constituent $90 \%$ de la consommation des pigments pour enregistrement magnétique.

La coercivité et l'aimantation rémanente des fines particules de $\gamma \mathrm{Fe}_{2} \mathrm{O}_{3}$ dérivent essentiellement de leur anisotropie de forme; c'est ce qui explique pourquoi les particules de $\gamma \mathrm{Fe}_{2} \mathrm{O}_{3}$ jusqu'à présent utilisées sont aciculaires : rapport d'acicularité de 6 à 10 , champ coercitif de 280 à 380 Oe, "carrure " (aimantation rémanente sur aimantation à saturation) entre 0,4 et 0,5 . Il est possible de renforcer la coercivité des particules de $\gamma \mathrm{Fe}_{2} \mathrm{O}_{3}$ en ayant un taux d'ions ferreux non nul (oxydes de Berthollide), mais ceux-ci sont moins stables que les oxydes non dopés.

L'intérêt pour l'enregistrement haute densité de particules isotropes à anisotropie multiaxiale, et la possibilité de renforcer l'anisotropie des particules par dopage par des ions d'anisotropie magnétocristalline élevée, est à l'origine du développement actuel d'une nouvelle catégorie de précurseurs $\gamma \mathrm{Fe}_{2} \mathrm{O}_{3}$ de faible acicularité.

$\gamma \mathrm{Fe}_{2} \mathrm{O}_{3}$ est métastable et ne peut être préparé directement à basse température (Fig. 5). Le pro- cédé le plus classique implique le passage par un oxyhydroxyde aciculaire [10]. Cet oxyhydroxyde est ensuite successivement déshydraté (obtention d'hématite $\alpha \mathrm{Fe}_{2} \mathrm{O}_{3}$ ), réduit (en magnétite $\mathrm{Fe}_{3} \mathrm{O}_{4}$ ) puis réoxydé pour donner $\gamma \mathrm{Fe}_{2} \mathrm{O}_{3}$. Ces différentes réactions sont pseudomorphiques, mais le réseau cristallin passe successivement du système orthorombique de $\alpha$ et $\gamma$ - $\mathrm{FeOOH}$, au système hexagonal de $\alpha \mathrm{Fe}_{2} \mathrm{O}_{3}$ puis au système cubique de $\mathrm{Fe}_{3} \mathrm{O}_{4}$ et $\gamma \mathrm{Fe}_{2} \mathrm{O}_{3}$ (spinelle lacunaire). Ce procédé, quoique très intéressant économiquement et couramment utilisé, présente deux inconvénients majeurs :

- la formation et la croissance des germes d'oxyhydroxyde est un procédé complexe et difficilement maîtrisable: malgré la somme des travaux dans le domaine (procédés basiques ou acides, utilisation de régulateurs de croissance), les particules présentent un grand nombre de dendrites et leur répartition en taille et en acicularité est trop large. (Noter que le procédé passant par $\gamma \mathrm{FeOOH}$ donne des particules plus aciculaires et moins accidentées que celles issues du procédé $\alpha \mathrm{FeOOH}$ mais ne permet pas d'utiliser des matières premières de récupération comme les sulfates).

- lors du passage de l'oxyhydroxyde à l'hématite $\alpha \mathrm{Fe}_{2} \mathrm{O}_{3}$, le départ d'eau entraîne une perte en poids de l'ordre de $10 \%$ et une microporosité importante. Les températures élevées des traitements oxydoredox ultérieurs permettent de réduire cette porosité (responsable de la création de champs démagnétisants internes et donc de l'abaissement de la coerci- 


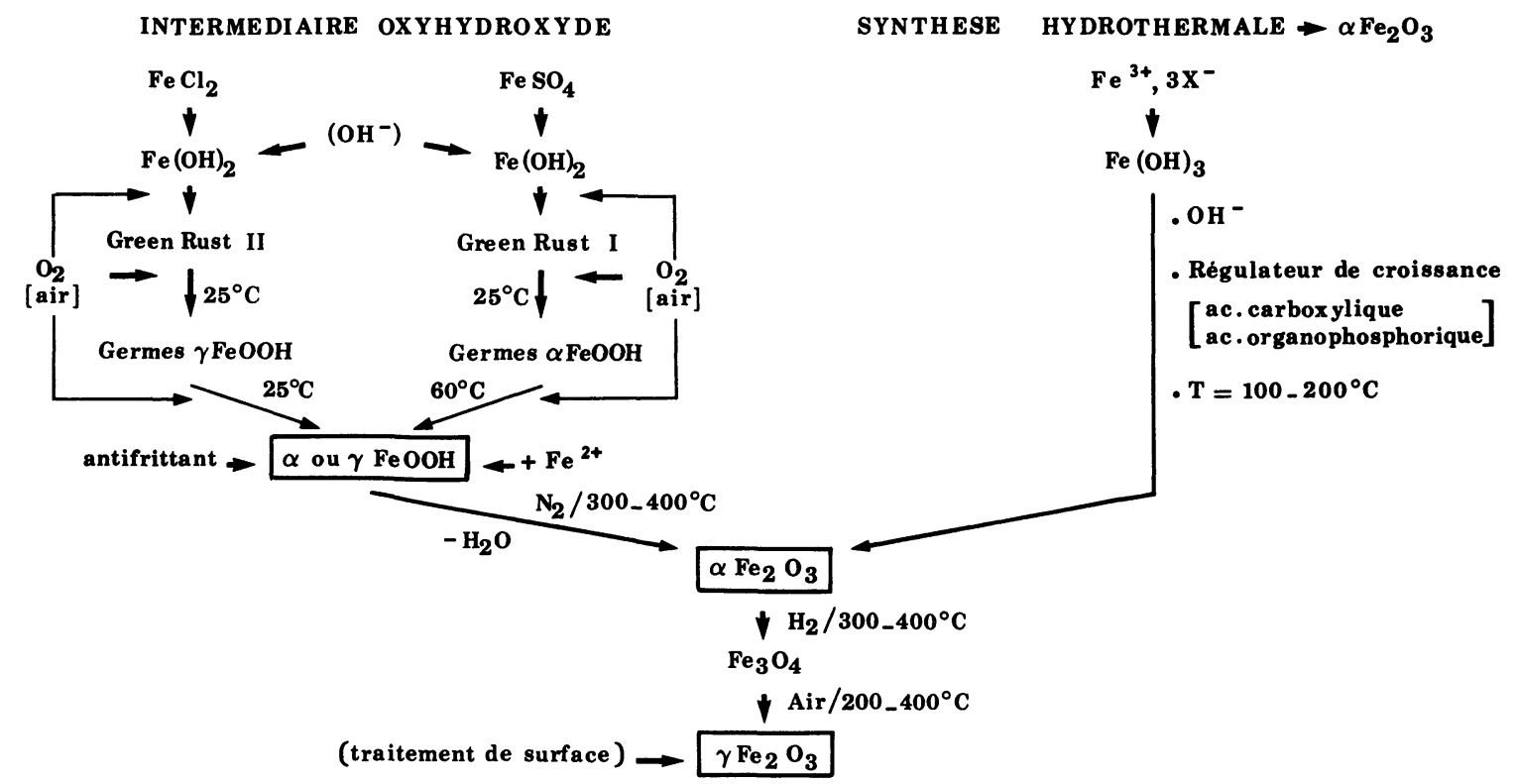

Fig. 6. - Méthodes de synthèse de $\gamma \mathrm{Fe}_{2} \mathrm{O}_{3}$.

[Production methods of $\gamma \mathrm{Fe}_{2} \mathrm{O}_{3}$.]

vité de la particule de $\gamma \mathrm{Fe}_{2} \mathrm{O}_{3}$ finale), mais entrâ̂nent des problèmes d'altération de l'acicularité, et de frittage des particules : le compromis est difficile à trouver, pour chaque type de précurseur, entre l'effet positif de la température (qui réduit la porosité et augmente $H_{\mathrm{c}}$ ) et son effet négatif (frittage, perte de l'acicularité des particules d'où abaissement de $H_{\mathrm{c}}$ et élargissement de la distribution des champs coercitifs ("Switching field distribution", SFD) [11].

L'intérêt de particules de faible porosité est à l'origine du procédé récemment introduit par Sakai $[12,13]$ pour l'élaboration de particules non poreuses (particules NP). Dans celui-ci, $\alpha \mathrm{Fe}_{2} \mathrm{O}_{3}$ est directe- ment produit par synthèse hydrothermale ; un précipité d'hydroxyde ferrique est maintenu plusieurs heures entre $100-200^{\circ} \mathrm{C}$, à des $\mathrm{pH}$ de $10-11$ en présence d'un agent régulateur de croissance. L'hématite obtenue est pratiquement homodisperse, non poreuse, et sans dendrite; sa taille et son acicularité peuvent être contrôlées entre $r=2$ à 10 , et $L=1$ à $0,1 \mu \mathrm{m}$. Outre leur faible porosité et donc la limitation des sources de désaimantation interne, les particules NP, sans dendrite, devraient permettre un meilleur compactage à l'intérieur de la couche.

Les clichés de microscopie électronique à transmission, de la figure 7 montrent l'évolution parcourue dans la morphologie des particules : meilleure uni-
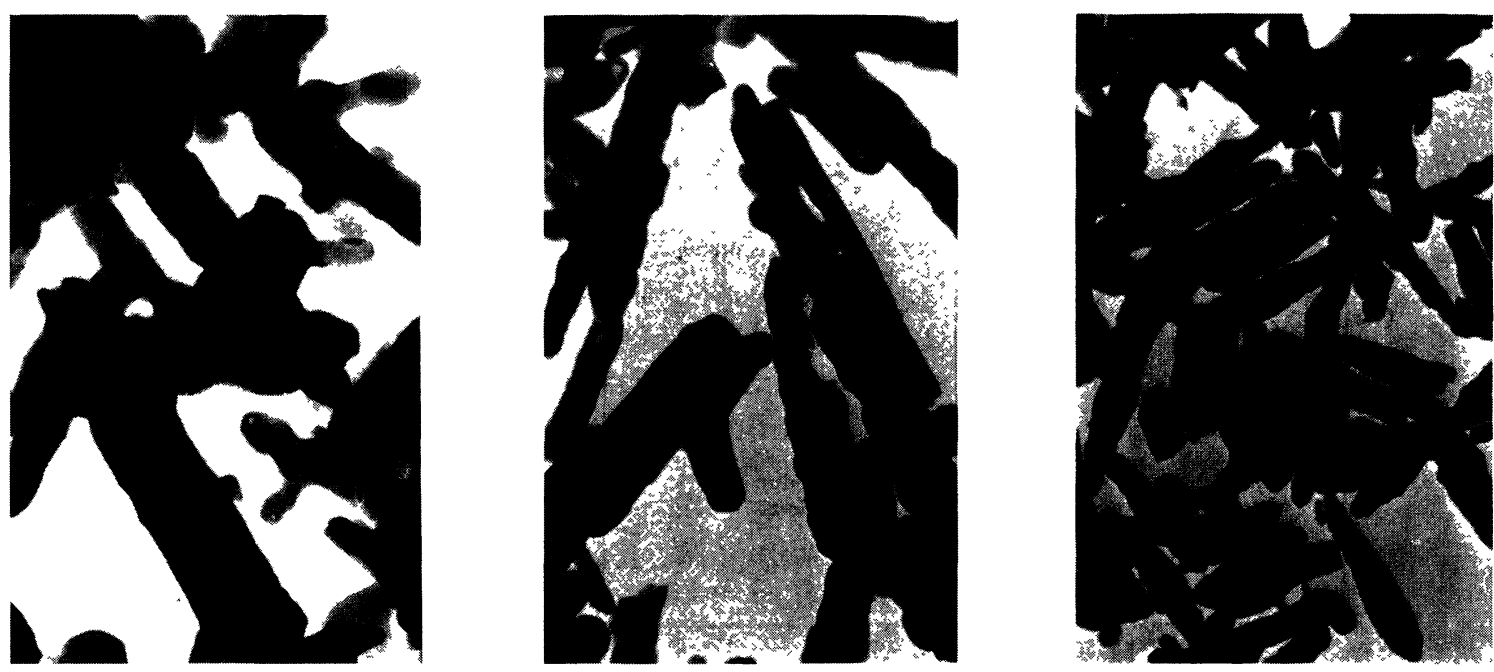

Fig. 7. - L'évolution des particules $\gamma \mathrm{Fe}_{2} \mathrm{O}_{3}$.

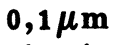

[The evolution of $\gamma \mathrm{Fe}_{2} \mathrm{O}_{3}$ particles.] 
formité, moins de branchements, particules plus fines.

Les particules de $\gamma \mathrm{Fe}_{2} \mathrm{O}_{3}$ ne sont pas monocristallines: elles sont constituées d'une mosaïque de particules élémentaires qui ont un axe cristallographique commun. Cet axe coïncide avec la direction de plus grande acicularité de la particule et constitue l'axe de facile aimantation [14]. Le mécanisme de retournement de l'aimantation dépend de la perfection de la morphologie et de la texture de la particule [15] : il serait incohérent du type fanning, pour le $\gamma \mathrm{Fe}_{2} \mathrm{O}_{3}$ classique, mais d'une autre nature non encore élucidée dans le cas des particules NP [13]. Il a été montré que la structure mosaïque et le mode de renversement de l'aimantation sont directement liés aux propriétés d'enregistrement, la référence [14] montre que le " print-through" (i.e. le transfert parasite de l'information d'une spire à une autre dans le cas des bandes) est d'autant plus important que le nombre d'entités élémentaires à l'intérieur de la particule est plus grand.

3.2 L'OXYDE DE FER DOPÉ COBALT' Co- $\gamma \mathrm{Fe}_{2} \mathrm{O}_{3}$. L'oxyde $\gamma \mathrm{Fe}_{2} \mathrm{O}_{3}$ modifié cobalt est après l'oxyde de fer standard, l'oxyde le plus utilisé. Il est employé dans les applications qui nécessitent des coercivités supérieures à $400 \mathrm{Oe}$ : bandes vidéo, disques souples haute densité.

Le principe est de renforcer, par l'introduction d'éléments dopants, l'anisotropie magnétocristalline des particules : selon la méthode de dopage, et le taux de cobalt, on peut ainsi obtenir des coercivités allant de 400 à plus de 1000 Oe.

La première génération d'oxydes modifiés cobalt est celle des oxydes dopés en volume, préparés par coprécipitation de sels de fer $^{2+}$ et de cobalt ${ }^{2+}[10]$. La présence des ions $\mathrm{Co}^{2+}$, distribués de manière aléatoire sur les sites $\mathrm{B}$ de la maille spinelle augmente la constante d'anisotropie magnétocristalline cubique. Du fait de la symétrie cubique du réseau, et de la répartition aléatoire des ions $\mathrm{Co}^{2+}$, il existe après couplage des spins électroniques des ions $\mathrm{Co}^{2+}$ et $\mathrm{Fe}^{3+}$, plusieurs directions de facile aimantation et la " carrure " (i.e. $\sigma_{\mathrm{r}} / \sigma_{\mathrm{m}}$ ) des oxydes dopés volume est élevée (carrure de 0,7 à $0,8)$ à comparer avec celles des particules uniaxiales, toujours inférieure à 0,5 .

Malheureusement, les oxydes fortement dopés en volume se sont avérés inutilisables pour l'enregistrement magnétique : la présence d'ions $\mathrm{Co}^{2+}$ rehausse la constante d'anisotropie magnétocristalline mais en augmente la dépendance vis-à-vis de la température et de ce fait celle de la coercivité et de la rémanence des particules.

$\mathrm{Si}$ on se souvient qu'un media magnétique doit pouvoir fonctionner entre 10 et $55^{\circ} \mathrm{C}$, et que des élévations de la température jusqu'à $60-70^{\circ} \mathrm{C}$ peuvent se produire à l'interface tête-media, on conçoit que la perte de 5 à 6 Oe des oxydes dopés volume (Fig. 8) est rédhibitoire : perte de niveau, déplacement du courant d'écriture, problèmes de surécriture (overwrite) si celle-ci est faite à haute température.

La présence de cobalt est aussi la cause de l'évolution dans le temps des paramètres magnétostatiques : la migration des ions cobalt dans la maille avec le temps de stockage, provoque une augmentation continue de la rémanence (phénomène exacerbé en présence de $\mathrm{Fe}^{2+}$ ) [18], ce qui peut induire des problèmes de print-through d'autant plus importants que la concentration en Co est plus élevée [19].
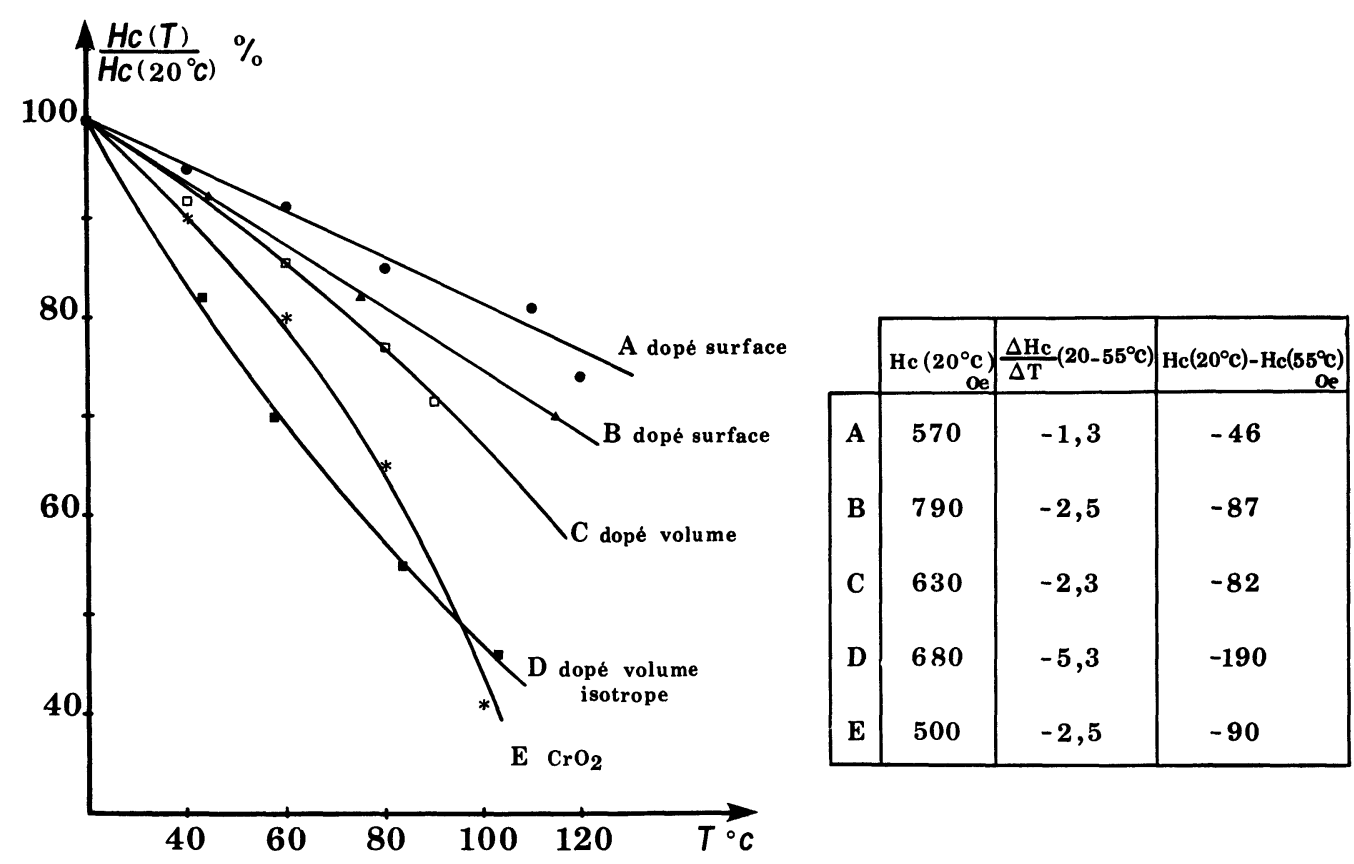

Fig. 8. - Dépendance thermique du champ coercitif.

[Temperature dependence of the coercivity.] 
Enfin la sensibilité aux contraintes mécaniques (magnétostriction), qui se traduit par une perte de signal avec le nombre de passages, augmente avec le taux de cobalt [20].

L'observation ayant été faite que l'adsorption de cobalt en surface permettait d'augmenter les coercivités tout en limitant la dépendance thermique, ont été introduits les oxydes modifés cobalt de la seconde génération: les oxydes dopés en surface. Trois méthodes de dopage ont été décrites dont seules les deux premières sont effectivement utilisées :

- dans la première méthode, seuls des ions $\mathrm{Co}^{2+}$ sont adsorbés (en milieu basique et à des températures modérées pour ne pas provoquer la migration du cobalt dans la masse de la particule) sur les particules précurseurs $\left(\gamma \mathrm{Fe}_{2} \mathrm{O}_{3}\right.$ ou oxydes de Berthollide). La coercivité augmente d'abord linéairement avec le taux de cobalt pour devenir optimale pour des taux voisins de $2 \%$. Passée cette valeur, le cobalt se retrouve sous la forme d'hydroxyde non magnétique et la coercivité décroît. Les coercivités optimales atteintes sont de 200 à 300 Oe supérieures à celles des précurseurs [21].

- la deuxième méthode consiste à adsorber simultanément du $\mathrm{Fe}^{2+}$ et du $\mathrm{Co}^{2+}$ en milieu basique et à des températures modérées. Il y a formation en surface d'un ferrite de cobalt (épitaxié) de composition proche de $\mathrm{CoFe}_{2} \mathrm{O}_{4}$ [22]. La coercivité augmente linéairement avec le taux de cobalt.

- la dernière méthode consiste à déposer en phase vapeur (sous mélange $\mathrm{N}_{2} / \mathrm{H}_{2}$ ) du cobalt sur des précurseurs $\mathrm{Fe}_{3} \mathrm{O}_{4}$ ou $\gamma \mathrm{Fe}_{2} \mathrm{O}_{3}$ fluidisés, le cobalt étant amené par la décomposition d'acétylacétonate de cobalt II (sublimable). La variation de la coercivité avec le taux de cobalt est très proche de celle obtenue avec la précédente méthode [23].

Les produits dopés en surface présentent une anisotropie magnétocristalline uniaxiale, l'axe de faible aimantation de la particule dopée se confond avec celui de la particule précurseur. Il semblerait que, sous l'effet du champ magnétique créé par le cœur de la particule, la couche riche en cobalt développe une anisotropie magnétocristalline superficielle uniaxiale, semblable à celle rencontrée dans les ferrites recuits sous champ, qui viendrait renforcer l'anisotropie du cour de la particule [24].

La figure 8 montre que la dépendance thermique des oxydes dopés cobalt en surface est bien plus faible que celles des dopés en volume, de 1 à $3 \mathrm{Oe} /{ }^{\circ} \mathrm{C}$ jusqu'à 7 à $8 \mathrm{Oe} /{ }^{\circ} \mathrm{C}$. On attribue ce phénomène à une plus grande stabilité vis-à-vis de la température de la constante d'anisotropie uniaxiale de surface comparée à celle de la constante d'anisotropie multiaxiale cubique. On constate aussi que la localisation en surface du $\mathrm{Co}^{2+}$, permet de limiter les pertes de signal par magnétostriction.

La présence de $\mathrm{Fe}^{2+}(5$ à $10 \%)$ permettant d'obtenir une coercivité donnée avec un taux de cobalt plus faible [23], du $\mathrm{Fe}^{2+}$ est souvent introduit pour minimiser la dépendance thermique des oxydes dopés cobalt. Malheureusement le $\mathrm{Fe}^{2+}$ privilégie la migration des ions $\mathrm{Co}^{2+}$ et augmente la dépendance dans le temps des propriétés magnétiques.

De nombreux travaux portent encore sur l'amélioration de la stabilité dans le temps, et vis-à-vis des contraintes thermiques et mécaniques des oxydes dopés cobalt en surface, à anisotropie uniaxiale : le blocage des lacunes de la maille spinelle destiné à limiter la migration des ions cobalt est une voie très étudiée.

Paradoxalement, ce ne sont pas les oxydes dopés en surface, mais les oxydes dopés en volume qui, en raison de leur capacité à donner une couche de carrure élevée dans toutes les directions, s'avèrent les plus attrayants pour l'enregistrement haute densité. Deux formes de particules sont possibles particules isotropes (cubiques, sphériques) ou particules de très faible acicularité $L / l=2$ (particules grains de riz dont le léger facteur de forme permet d'utiliser moins de cobalt pour atteindre une coercivité donnée). Des valeurs de D 50 de 65000 bpi (D50 = valeur de la densité à laquelle le niveau de sortie vaut $50 \%$ de sa valeur maximale) ont été décrites pour les oxydes dopés isotropes contre 45000 bpi au maximum pour les oxydes dopés aciculaires [4,5]. Pour que la voie « dopé cobalt isotrope » réussisse, il reste à montrer qu'il est possible de limiter les coefficients de magnétostriction et de dépendance thermique de la coercivité de ces particules ce qui n'est pas évident comme le montrent les problèmes de développement du disque Isomax de Kodak.

3.3 LE FER MÉTAL. - Les particules de fer ont, par rapport aux particules d'oxydes, de nombreux atouts. (i) Le fer pur a une aimantation à saturation et une densité absolue bien plus élevées $\left(\sigma_{\mathrm{s}}=\right.$ $210 \mathrm{uem} / \mathrm{g}$ et $\rho=7-8 \mathrm{~g} / \mathrm{cm}^{3}$ comparativement à $\sigma_{\mathrm{s}}=74 \mathrm{uem} / \mathrm{g}$ et $\rho=5 \mathrm{~g} / \mathrm{cm}^{3}$ ), ce qui permet des aimantations rémanentes par unité de volume de couche importantes. (ii) Les coercivités des fines particules sont larges, d'où une moindre tendance à la désaimantation même à haute densité. En outre, (iii) il est possible de réaliser des particules de fer, stables vis-à-vis du superparamagnétisme (pas de phénomène de spin canting pour le fer pur [9]), dans des gammes de tailles sensiblement plus faibles que celles autorisées avec les oxydes $(50 \mathrm{~m} 2 / \mathrm{g}$ contre $40 \mathrm{~m} 2 / \mathrm{g}$ selon [25]), d'où un plus grand nombre de particules par unité de volume et une dynamique signal/bruit élevée. Cependant, la manipulation de fines particules de fer pose de nombreux problèmes et l'on est loin de savoir tirer parti de toutes leurs potentialités.

3.3.1 La tendance à l'oxydation des fines particules de fer est bien connue : la pyrophoricité des particules ne peut être réduite que par passivation superficielle (si possible par une couche magnétique, passivation oxyde) ou par transformation en un matériau magnétique, stable, d'aimantation proche du fer (transformation en $\mathrm{Fe}_{4} \mathrm{~N}, \sigma_{\mathrm{s}}=160 \mathrm{uem} / \mathrm{g}$ [26]). Dans tous les cas, les méthodes actuelles de passivation ne permettent d'atteindre que des aimantations 
très inférieures à celles du fer pur, et les produits sont bien moins stables que les oxydes standards (perte de $\sigma_{\mathrm{s}}$ à $60{ }^{\circ} \mathrm{C}, 90 \% \mathrm{RH}$ [26]).

3.3.2 la densité absolue des particules obtenues à partir des précurseurs oxyhydroxyde (voie la plus utilisée) est loin de la densité théorique : la porosité interne est importante, elle correspond à une perte en poids de l'ordre de $40 \%$ entre l'oxyhydroxyde initial et la particule de fer.

D'autre part, le développement des particules de fer a été retardé par leur incompatibilité avec les têtes d'enregistrement classiques en ferrites, celles-ci saturant au-dessus de 1000 Oe, valeur de coercivité communément obtenue avec les particules de fer métal aciculaires. Une première génération de particules de fer a donc été développée, dont la gamme de coercivité est contenue entre 600 et 900 Oe par contrôle de leur acicularité et/ou dopage $\mathrm{Ni}$. Ces particules testées en audio et vidéo, se sont montrées bien supérieures aux oydes $\mathrm{Co}-\gamma \mathrm{Fe}_{2} \mathrm{O}_{3}$ de mêmes coercivités [27]. Les particules à 1 400-1500 Oe, ne connaissent qu'un développement tout récent dans la vidéo $8 \mathrm{~mm}$, grâce aux progrès accentués dans la technologie des têtes amorphes. La courbe de réponse en fréquence de la figure 9 (Réf. [34]) montre l'intérêt du fer aciculaire orienté par rapport aux particules dopées cobalt classiques.

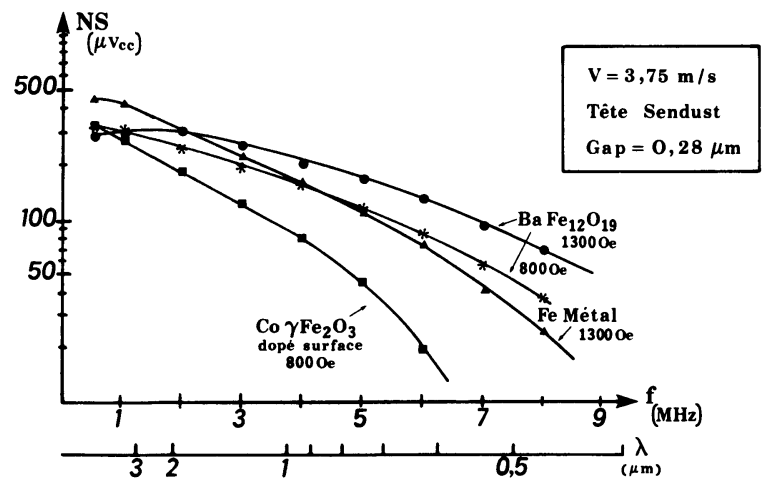

Fig. 9. - Réponse en fréquence de différents types de bandes.

[Frequency response of different tapes.]

Les méthodes de synthèse des particules de fer sont multiples [28], les particules obtenues sont plus ou moins poreuses selon la méthode, mais le problème de leur stabilité lui, se pose toujours in fine. La voie la plus communément utilisée est la réduction par l'hydrogène et éventuellement la nitruration de précurseurs oxydes ou oxyhydroxydes. Les particules de fer gardent la morphologie de leur précurseur mais sont fortement poreuses $(65-75 \%$ de porosité en volume selon [29]) : divers dopages ou traitements antifrittants sont utilisés pour empêcher la coalescence des particules, maintenir leur acicularité et diminuer leur porosité [30]. Les particules sont nettement polycristallines (cf. Fig. 10) ; à taille

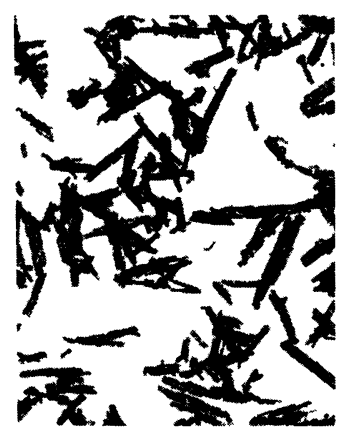

$\stackrel{0,3 \mu \mathrm{m}}{ }$

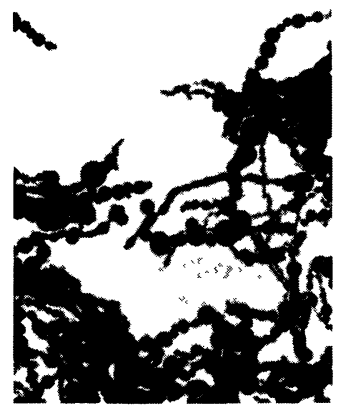

$0,2 \mu \mathrm{m}$
Fig. 10. - Particules de fer métal.

[Iron particles.]

et forme de précurseur données, leur coercivité dépend étroitement de la taille des microcristallites, elle est maximale pour des cristallites de 200-300 $\AA$ (cristallite monodomaine).

Une autre voie consiste à préparer les particules de fer par décomposition et réduction de précurseurs aciculaires d'oxalates ferreux [31] $(L=5 \mu \mathrm{m})$. Dans le cas présent, la matrice du précurseur est complètement pulvérisée en particules de formes et de tailles aléatoires, enchaînées les unes aux autres. L'étroite dépendance de la coercivité de la particule vis-à-vis de la taille des microcristallites et la difficulté de maîtriser l'éclatement de la particule font qu'il est difficile d'avoir des expériences reproductibles et d'obtenir des répartitions de champs coercitifs resserrées.

Plus coûteuses et peu utilisées sont les élaborations par réduction de sels ferreux par du borohydrure de sodium [32] ou la condensation sous vide de vapeurs métalliques [33]. Les particules se présentent sous forme de petites billes, monodomaines de $100-300 \AA$, accolées les unes aux autres sous forme de chaînes (cf. Fig. 10). La méthode de condensation sous vide, semblerait donner des particules de meilleure cristallinité moins sensibles à l'oxydation.

La coercivité et la rémanence des particules de fer métal dérivent essentiellement de son anisotropie de forme : anisotropie de forme du précurseur oxyde ou de la chaîne de billes selon la méthode d'élaboration.

3.4 L'OXYDE DE CHROME $\mathrm{CrO}_{2}$. - L'oxyde de chrome a été introduit par Du Pont à la fin des années 60 pour prendre le relais des oxydes $\gamma \mathrm{Fe}_{2} \mathrm{O}_{3}$ de basse coercivité [35]. Il est, depuis en concurrence avec les oxydes dopés cobalt.

N'existant pas à l'état natif dans la nature, $\mathrm{CrO}_{2}$ est préparé dans des conditions hydrothermales par oxydation de $\mathrm{Cr}_{2} \mathrm{O}_{3}$ par un excès de $\mathrm{CrO}_{3}$ (cf. Fig. 11). La décomposition du $\mathrm{CrO}_{3}$ en excès libère de l'oxygène qui sous pression, stabilise $\mathrm{CrO}_{2}$ vis-àvis de l'hydrolyse $\left(\rightarrow \mathrm{Cr}^{\mathrm{IV}}+\mathrm{Cr}^{\mathrm{III}}\right)$. Une étape intermédiaire de passivation par réduction de la surface en hydroxyde de $\mathrm{Cr}^{\mathrm{III}}$ est nécessaire avant obtention du produit définitif. 


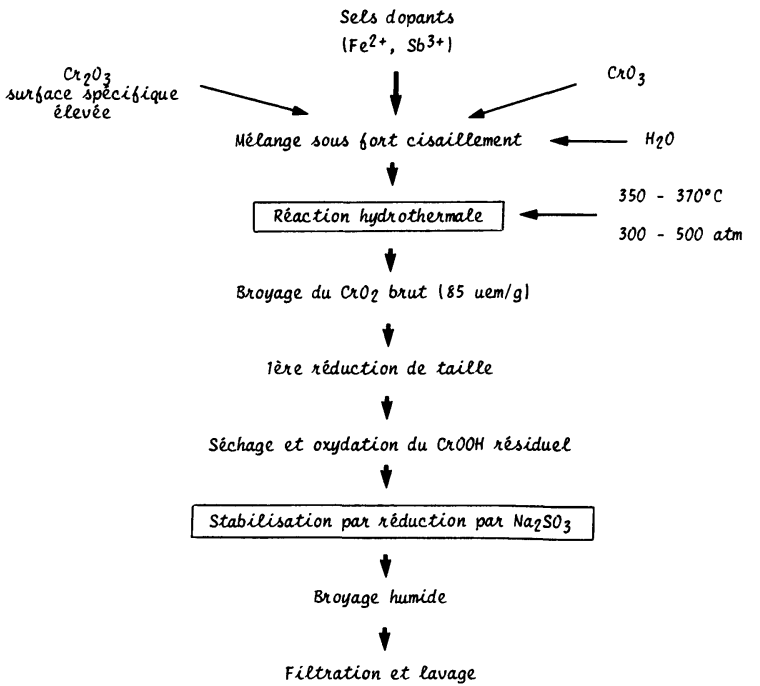

Fig. 11. - Synthèse de $\mathrm{CrO}_{2}$.

[Production methods of $\mathrm{CrO}_{2}$.]

Les particules obtenues se présentent sous forme de fines aiguilles monocristallines non poreuses, très bien définies (Fig. 12). $\mathrm{CrO}_{2}$ est ferromagnétique et sa coercivité dérive essentiellement de son anisotropie de forme. Des coercivités de 400 à 670 Oe peuvent être obtenues par dopage par des ions $\mathrm{Fe}^{3+}$ ou $\mathrm{Sb}^{3+}$, dont le rôle est à la fois d'agir comme agent nucléant (augmentation de la surface spécifique de 15 à $60 \mathrm{~m}^{2} / \mathrm{g}$ avec quelques \% de dopants) et de renforcer l'acicularité des particules [36]. La substitution du $\mathrm{Cr}^{\mathrm{IV}}$ par des ions $\mathrm{Ir}^{\mathrm{IV}}$ ou $\mathrm{Rh}^{\mathrm{IV}}\left(\mathrm{IrO}_{2}\right.$ et $\mathrm{RhO}_{2}$ cristallisent comme $\mathrm{CrO}_{2}$ dans la maille rutile) permet d'augmenter encore la coercivité par renforcement de l'anisotropie magnétocristalline [37]. Passivation et dopage ont pour conséquence de redescendre l'aimantation à saturation de $\mathrm{CrO}_{2}$ de sa valeur théorique de $100 \mathrm{uem} / \mathrm{g}$ à celles de $73-78 \mathrm{uem} / \mathrm{g}$.

L'oxyde de chrome a par rapport aux oxydes dopés cobalt, l'avantage de cristalliser sous forme de monocristallites très bien définies. On peut obtenir ainsi des couches extrêmement compactes et bien orientées (carrure $\mathrm{Br} / \mathrm{Bm}$ et rapport d'orientation $\mathrm{Br} / / / \mathrm{Br}_{\perp}$ élevées). Cet atout se retourne néanmoins contre le $\mathrm{CrO}_{2}$ quand on vise une application aux disques pour informatique où on cherche à désorienter les particules). D'autre part, $\mathrm{CrO}_{2}$ étant conducteur, on évite de charger la couche en carbone aux dépends de la charge magnétique et du liant. Son principal travers est la valeur relativement faible de son point de Curie, ce qui induit des variations relativement importantes de la coercivité dans la gamme de température utilisée en enregistrement magnétique $\left(10-55^{\circ} \mathrm{C}\right)$ (cf. Fig. 8).

Utilisé jusqu'à présent dans les applications vidéo et audio (sauf au Japon) l'oxyde de chrome connaît un regain d'intérêt en informatique avec l'introduction sur le marché de la cartouche IBM pour systèmes 3480 (25000 bpi).

Mais du fait de son caractère uniaxial, et de la valeur limitée de sa coercivité $\mathrm{CrO}_{2}$ ne paraît pas le mieux placé dans la course aux hautes densités.

3.5 L'HEXAFERRITE DE BARYUM. - L'enregistrement perpendiculaire permet d'augmenter les densités d'enregistrement tout en limitant les risques de désaimantation bit à bit. Parmi les solutions technologiques possibles, les films Co-Cr et Terres RaresFer très intéressants potentiellement, n'ont que fort peu de chances de pouvoir être produits massivement et à moyen terme, et ce sont les particules d'hexaferrite de baryum qui sont le mieux placées pour permettre avec les moyens d'enduction actuels, la production en masse de supports d'enregistrement perpendiculaire. L'originalité des particules d'hexaferrite $\mathrm{BaFe}_{12} \mathrm{O}_{19}$ est de se présenter sous la forme de plaquettes hexagonales (Fig. 13) d'avoir une anisotropie magnétocristalline importante et une direction de facile aimantation perpendiculaire aux plans des plaquettes [38, 39]. Il est alors beaucoup plus facile d'orienter les aimantations de ces plaquettes perpendiculairement au plan de la couche (en apposant par exemple un champ magnétique perpendiculaire pendant toute la durée du séchage de l'enduit), que d'essayer d'aligner perpendiculairement à la couche des particules aciculaires uniaxiales.

Les méthodes d'élaboration bien connues par chamottage (calcination à haute température de mélanges de carbonates de baryum et d'oxydes de fer, puis broyage) utilisées pour les hexaferrites pour aimants permanents ne sont malheureusement pas adaptées aux particules pour enregistrement magné-
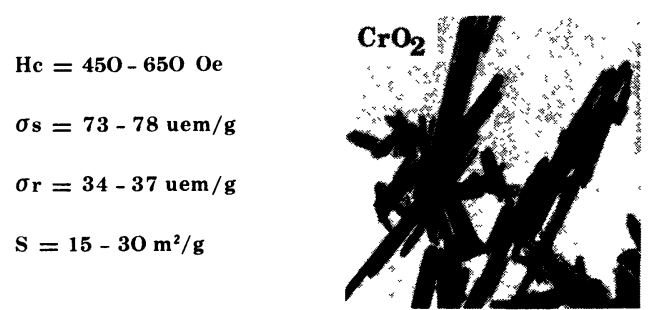

Fig. 12. - Particules d'oxyde de chrome.

$\left[\mathrm{CrO}_{2}\right.$ particles. $]$
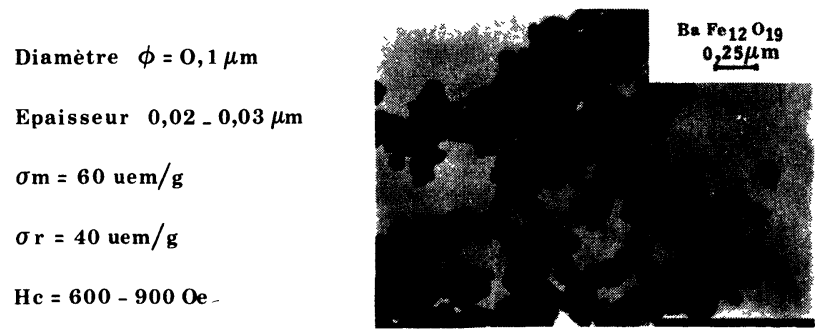

Fig. 13. - Particules d'hexaferrite de baryum.

[Baryum hexaferrite particles.] 
tique : les particules doivent être fines $(0,1$ contre 1 à plusieurs $\mu \mathrm{m})$, non frittées, de répartition granulométrique resserrée.

En outre, pour des raisons de compatibilité avec les têtes d'enregistrement, la coercivité des hexaferrites pour enregistrement magnétique doit être inférieure à $1000 \mathrm{Oe}$ (têtes ferrites) ou $1600 \mathrm{Oe}$ (têtes amorphes), il est nécessaire de diminuer l'anisotropie magnétocristalline des particules par dopage par différents éléments, le plus souvent le couple $\mathrm{Co}-\mathrm{Ti}$ [40] (des particules non dopées de $0,1 \mu \mathrm{m}$ ont un champ coercitif de l'ordre de $5000 \mathrm{Oe}$ ). La difficulté est de ne pas trop réduire lors du dopage, l'aimantation à saturation de $\mathrm{BaFe}_{2} \mathrm{O}_{19}$ déjà faible $(60 \mathrm{uem} / \mathrm{g})$.

Différentes synthèses sont développées, le problème majeur étant d'obtenir des particules homodisperses et non frittées [41] :

- La méthode des vitro-céramiques (Toshiba [40]) : un mélange d'oxyde de baryum $\mathrm{BaO}$, d'hématite $\alpha \mathrm{Fe}_{2} \mathrm{O}_{3}$, d'anhydride borique $\mathrm{B}_{2} \mathrm{O}_{3}$ contenant un faible pourcentage d'oxyde de cobalt et de titane est fondu puis trempé brusquement entre deux rouleaux d'acier. Le verre au bore ainsi obtenu est recuit et les particules d'hexaferrites cristallisées sont ensuite séparées de la matrice vitreuse par attaque à l'acide acétique.

- La synthèse hydrothermale à partir de solutions contenant des sels de $\mathrm{Ba}^{2+}, \mathrm{Co}^{2+}$ et $\mathrm{Ti}^{2+}$ et un précurseur de $\mathrm{Fe}$ (sel ferrique ou précurseur solide $\left.\alpha \mathrm{Fe}_{2} \mathrm{O}_{3}, \alpha-\gamma \mathrm{FeOOH}\right)$ [43].

- La méthode de précipitation-recuit : des sels de fer et de baryum sont précipités en milieu très basique puis recuits à haute température [43].

Les performances des hexaferrites, à courte longueur d'onde, sont excellentes :

- La figure 9 (issue de la Réf. [34]) montre que même avec une tête à anneau classique, les hexaferrites orientés (aimantation perpendiculaire au plan de la couche) permettent des densités beaucoup plus élevées que les oxydes dopés cobalt en surface ou le fer métal. L'utilisation de têtes unipolaires, spécialement conçues pour l'enregistrement perpendiculaire, permet encore d'améliorer ces résultats [39].
- Des bandes, réalisées sans orienter magnétiquement les particules d'hexaferrites (media « pseudoisotrope "), sont elles aussi supérieures à celles réalisées avec des particules métal [34].

Les hexaferrites de baryum sont donc très intéressants pour les applications haute densité, des améliorations devront cependant être apportées concernant : (i) l'obtention de particules fines de granulométrie resserrée, (ii) le contrôle de leur variation en température (augmentation de $H_{c}$ quand la température augmente au voisinage de l'ambiante), (iii) leur facilité de mise en dispersion (les particules ont tendance à s'agglutiner les unes aux autres).

\section{Conclusion.}

Les densités d'enregistrement peuvent encore être augmentées par utilisation de milieux d'enregistrement magnétique particulaires.

La technique d'enregistrement magnétique sur film mince donne d'excellentes performances électriques, qui sont néanmoins inférieures à celle de l'enregistrement optique, qu'elle a donc peu de chances de concurrencer à long terme. Les problèmes de prix de revient, de tenue mécanique et de corrosion du media, constituent aussi un frein au développement à moyen terme des films minces magnétiques, et ce au profit de l'enregistrement sur media particulaire.

Dans ce dernier domaine, deux types de techniques peuvent permettre une augmentation des densités d'enregistrement :

- enregistrement longitudinal sur des particules orientées, de coercivité élevée (fer métal passivé),

- enregistrement isotrope ou perpendiculaire (fer métal, hexaferrites de baryum, oxydes dopés cobalt isotropes).

A l'heure actuelle, il paraît difficile de dire quelle est la technique qui prévaudra dans 10 ans, même si $a$ priori, l'hexaferrite de baryum semble le plus intéressant, de nombreux facteurs peuvent en effet bouleverser la hiérarchie actuelle des particules.

\section{References}

[1] WHITE, R. M., IEEE Spectrum 20 (1983) 32.

[2] IwASAKI, S., OUCHI, K., IEEE Trans. Magn. MAG 14 (1978) 849.

[3] Speliotis, D. E., IEEE Trans. Magn. MAG 20 (1984) 75

[4] LeMKe, J. E., IEEE Trans. Magn. MAG 15 (1979) 1561 .

[5] Giraud, A., Journées d'Etudes Mémoires Magnétiques, Grenoble, Juin 1985.

[6] FujiwARA, T., Intermag. 85, IEEE Trans. Magn. (sous presse).

[7] Kryder, M. K., J. Appl. Phys. 57 (1985) 3913.

[9] Morrish, A. H., HANEdA, K., J. Mag. Mag. Mat. 15-18 (1980) 1089.
[10] BATE, G., Ferromagnetic Materials Vol. 2, chapitre 7, (édité par North-Holland Publishing Company) 1980.

[11] Corradi, A. R., Mello Ceresa, E., IEEE Trans. Magn. MAG 15 (1979) 1068.

[12] Matsumoto, S., Koga, T., Fukai, K., NaKatani, S., US Patent $n^{\circ} 4,202,871,13$ mai 1980.

[13] Corradi, A. R., ANDress, S. J., French, J. E., Bottoni, G., Candolfo, D., CeCCheti, A. MASOLI, F., IEEE Trans. Magn. MAG 20 (1984) 33.

[14] Andress, S. J., Benedetti, A., Corradi, A. R., FAGHERAZZI, G., Intermag. 85, IEEE Trans. Magn. (sous presse). 
[15] Knowles, J. E., IEEE Trans. Magn. MAG 20 (1984) 84.

[16] Bickford, L. R., Brownlow, J. M., Penoyer, R. F., Proc. Inst. Elec. Eng. 104B (1957) 238, suppl. $\mathrm{n}^{\circ} 5$.

[17] Slonczewski, J. C., Phys. Rev., 110 (1958) 1341.

[18] Kishimoto, M., IEEE Trans. Magn. MAG 15 (1979) 906.

[19] Hayama, F., Functional Mat., 6 (1982) 47.

[20] Flanders, P. F., Kaganowicz, G., TAKeI, Y., IEEE Trans. Magn., MAG 15 (1979) 1065.

[21] IMAOKA, Y., UNEKI, S., KUbUTA, Y., TOKUOKA, Y. IEEE Trans. Magn. MAG 14 (1978) 649.

[22] Kishimoto, M., Sueyoshi, T., Hirata, J., AmemiYa, M., Hayama, J. Appl. Phys. 50 (1979) 450.

[23] Monteil, J. B., Dougier, P., Ferrites Proc. Int. Conf., Octobre 80, Kyoto Japan, p. 532.

[24] Penoyer, R. F., Bickford, L. R., J. Phys. Rev., 108 (1957) 271

[25] Corradi, A. R., MMA Grenoble, juin 1985.

[26] SuZuKi, S., SAKumoto, H., OMOTE, Y., MiNEGISHI, J., IEEE Trans. Magn. MAG 20 (1984) 48.

[27] Suzuki, S., SaKumoto, H., Miya, S., Omote, Y., MINEGISHI, J., IEEE Trans. Magn. MAG 20 (1984) 39.

[28] AKASHI, G., Proc. Int. Conf. Ferrites, octobre 80, Kyoto Japan, p. 548.

[29] SuzuKI, S., OMOTE, Y., MiYA, S., Yoshida, I., IshidA, A., Minegishi, J., Iwatare, O., Proc. Int. Conf. Ferrites, Kyoto Japan, octobre 80, p. 556.
[30] Sueyoshi, T., Naono, H., Kawanami, M., AmeMIYA, M., HAYANA, H., IEEE Trans. Magn. MAG 20 (1984) 42.

[31] SuZuki, S., SAKumoto, H., Minegishi, J., OMOTE, Y., IEEE Trans. Magn. MAG 17, (1981) 3017.

[32] Oppegard, A. L., Darnell, F. F., Miller, H. C. J. Appl. Phys. 32 (1961) 1845.

[33] TASAKI, A., SAegusa, N., OdA, M., IEEE Trans. Magn. MAG 19 (1979) 1731.

[34] FuJiwaRA, T., Intermag. 85, IEEE Trans. Magn. (sous presse).

[35] Chen, H. Y., Miller, D. M., Hudson, J. E., Wes TENBROEK, C. J. A., IEEE Trans. Magn. MAG 20 (1984) 24.

[36] Ingraham, J. N., Swaboda, T. J., US Patent 3 , 034, 988, 15 mai 1962.

[37] Maestro, P., Andriamandroso, D., Demazeau, G., Pouchard, M., Hagenmuller, P., IEEE Trans. Magn. MAG 18 (1982) 1000.

[38] FuJiwara, T., IsshiKI, M., KOIKE, Y., OGUCHI, T., IEEE Trans. Magn. MAG 18 (1982) 1200.

[39] Yokoyama, H., dans Recent Magnetics for Electronics (édité par North Holland Publishing Company) 1984, p. 187-198.

[40] Kubo, O., IDo, T., YokoYama, M., IEEE Trans. Magn. MAG 18 (1982) 1122.

[41] Hibst, H., Angew. Chem. Int. Ed. Engl. 21 (1982) 270.

[42] Takada, T., Kiyama, M., Ferrites Int. Conf. Kyoto, (1971) 380.

[43] Haneda, K., Kojima, H., J. Amer. Ceram. Soc. 57 (1974) 354. 